\title{
Aplicación de la dimensión identidad de la entrevista STIPO para diagnosticar difusión de identidad en pacientes con diagnóstico previo de trastornos severos de la personalidad. Estudio clínico cuantitativo
}

\author{
Application of identity dimension in the STIPO interview: \\ Diagnosis of identity difusion in patients with pevious \\ diagnosis of severe personality disorder. \\ A quantitative clinical study
}

Roberto F. Castillo T. ${ }^{1}$ y Helga M. Arias Z. ${ }^{2}$

\begin{abstract}
Scale study that applied STIPO (Structured Interview of Personality Organization - Structured Interview of Personality Organization) in Identity Dimension, 61 patients with and without a diagnosis of personality disorder according to DSM-IV, with the assess Identity versus identity diffusion Integrated. This research was based on the model of Otto Kernberg, where it is considered that the Identity is the central criterion in assessing the Personality Organization. This paper will expose the main theoretical and clinical foundations in reference to the Identity of the Self of this author. According to the results obtained in the sample, there is a high degree of concordance between the diagnosis of Personality Disorder according to DSM-IV diagnosis of Identity Diffusion through STIPO Identity Dimension.
\end{abstract}

Key words: Identity, Structural Diagnosis, Personality Disorders.

Rev Chil Neuro-Psiquiat 2018; 56 (1): 9-17

\section{Introducción}

$\mathrm{L}$ a evaluación clínica de los Trastornos Severos de la Personalidad, constituye un tema relevante para la investigación contemporánea, tanto desde el punto de vista diagnóstico como en el diseño de estrategias de abordaje más eficientes. En ella, cobra crucial preponderancia la evaluación de la
Identidad del Yo al constituirse como el elemento central a la hora de distinguir entre presentar o no un Trastorno Severo de la Personalidad.

Esta investigación se inició en el año 2012, empleando el DSM IV ${ }^{1}$ como vía diagnóstica para diagnosticar la presencia o no de Trastorno de Personalidad y, por otra parte, utilizamos la sección Identidad de la prueba STIPO$^{7}$ a fin de comprobar

Recibido: $31 / 08 / 2017$

Aprobado: 08/03/2018

Los autores no presentan ningún tipo de conflicto de interés.

1 Psiquiatra Adultos. Departamento de Psiquiatría. Escuela de Medicina. Universidad de Valparaíso, Hospital del Salvador Valparaíso.

2 Psicóloga. Hospital del Salvador Valparaíso. 
si era posible llegar a diagnosticar Identidad Integrada versus Difusión de Identidad únicamente utilizando la sección: Dimensión de la Identidad de la STIPO, esto basándonos en dos aspectos, por un lado el soporte teórico clínico que el Dr. Otto Kernberg y su equipo dan a la Identidad del Yo como elemento diagnóstico prioritario ${ }^{2-6} \mathrm{y}$, por otra parte, el sustento estadístico obtenido al evaluarse la capacidad psicométrica de la STIPO, donde se determinó que la Dimensión Identidad y la Dimensión Defensas Primitivas mostraron una consistencia interna adecuada, siendo estos dos dominios los que están estrechamente vinculados a la presencia de un Trastorno Severo de la Personalidad ${ }^{8}$.

En nuestro país, en el año 2008, se realizó una investigación abocada a la traducción y validación del Instrumento Structured Interview of Personality Organization (STIPO). En dicho estudio, tanto la confiabilidad del instrumento general como cada una de las dimensiones, arrojaron valores significativos, donde la Dimensión de Identidad logró un coeficiente de confiabilidad de 0,93 lo cual le otorga alto peso estadístico; más recientemente, en el 2013, se realizó en Alemania la validación a la versión Germana de la STIPO donde todas las dimensiones fueron significativas ${ }^{10}$.

Con estos antecedentes pretendimos solo utilizar la Dimensión Identidad de la STIPO con el fin de someterla a su especificidad y efectividad clínica a la hora de evaluar por sí sola la Dimensión Identidad.

Además, quisimos probar si dicho ítem guardaba alguna relación estadística o concordancia con la evaluación clínica previa que tenían nuestros pacientes y que consistía en tener o no tener Trastorno Severo de la Personalidad según DSM IV, lo cual conforme el modelo de Otto Kernberg implica que los pacientes tendrían o no un Trastorno en la Identidad Del Yo (Difusión de la Identidad vs Identidad Integrada).

\section{Identidad del yo}

Otto Kernberg estableció que la Difusión de Identidad es la piedra angular para realizar la distinción de Organización de Personalidad Limítrofe de la Organización de Personalidad Neurótica ${ }^{2-6}$.
Kernberg, plantea que el concepto de Identidad del Yo se representa en términos de relaciones de objeto y corresponde al nivel más alto de la organización de los procesos de internalización, que ocurre bajo el principio orientador de la función sintética del Yo. La formación de la Identidad es un proceso intrapsíquico, integracional de representaciones del sí mismo y de representaciones de otros significativos, los cuales son investidos, libidinal y agresivamente, de una manera cohesionada e integrada, proceso que conduce a la conformación de un sí mismo y de objetos totales.

La Identidad Integrada consiste en la presencia de un concepto integrado del sí mismo a través del tiempo y de situaciones diferentes, y un concepto integrado de personas significativas en su vida, la cual es propia de la Organización de Personalidad de tipo Neurótica.

Kernberg y colaboradores, realizaron un gran aporte al determinar que la presencia de Difusión de Identidad caracteriza a todos los Trastornos Severos de Personalidad agrupados bajo la denominada Organización de Personalidad Limítrofe. La Difusión de Identidad implica modelos de trabajo interno que reflejan la representación desorganizada/desorientada del sí mismo y los otros, es decir, conceptos pobremente integrados de ambos. Lo anterior se produce por la primacía de los mecanismos de escisión que fragmentan lo representativo del sí mismo y las representaciones de los otros en términos de disposiciones afectivas polares de extremos opuestos. Clínicamente, esto se evidencia en una experiencia subjetiva de vacío crónico, autopercepciones y conductas contradictorias que no pueden integrarse en una forma emocionalmente significativa y, por otro lado, con percepciones superficiales, insípidas y empobrecidas de los demás ${ }^{2-6}$.

La falta de integración de los aspectos buenos y malos de la realidad de sí mismo y de los otros, se debe a la predominancia de una agresión temprana activada en estos pacientes, donde las introyecciones e identificaciones generadas bajo la influencia de derivados de instintos libidinales, se constituyen totalmente separados de los derivados de instintos agresivos, manteniéndose separados y divididos (escindidos) por la falta de capacidad sintética del 
Yo, dichos polos son utilizados, posteriormente, de manera defensiva para impedir el surgimiento de ansiedad y de esta manera proteger al núcleo Yoico, acción primordial de los mecanismo basados en la escisión ${ }^{2-6}$.

La incapacidad para sintetizar las introyecciones e identificaciones buenas y malas que ocurren en momentos de Afectos Cumbre, interfiere con la normal modulación y diferenciación de las disposiciones afectivas del Yo y condiciona la irrupción de estados afectivos primitivos. Por otra parte, esta falta de integración de los derivados de instintos libidinales y agresivos, interfiere en la capacidad yoica de poder elaborar preocupación y culpa, generando además, serios obstáculos en la integración del Súper Yo, ya que, los precursores superyoicos sádicos son demasiado intensos para ser tolerados, ante lo cual son reproyectados como objetos externos transformándose en objetos malos y persecutorios. De esta manera se crean imágenes del sí mismo e imágenes objetales parciales, es decir, totalmente idealizadas que forman ideales desproporcionados de poder, perfección y grandeza, o imágenes totalmente devaluadas o malas, lo cual, imposibilita el poder percibir a los otros y a sí mismo de manera realista e integrada ${ }^{1,3,4-6,11}$.

Kermberg y su equipo diseñaron La Entrevista Estructural la cual es un método muy completo y eficiente que permite realizar un diagnóstico psiquiátrico clásico, determinar el tipo de Organización de Personalidad, el tipo particular de Trastorno de Personalidad y los diagnósticos dinámicos y epigenéticos. Permite, además, evaluar la capacidad y motivación del paciente para ser tratado con psicoterapia; estos aspectos a evaluar requieren de varias sesiones de entrevistas diagnósticas ${ }^{5,6}$. Con la intención de hacer sustentable la Entrevista Estructural a la investigación empírica, en el año 2006, elaboraron la Entrevista Estructurada de Organización de la Personalidad (STIPO) $)^{7}$ instrumento altamente específico y de alta confiabilidad ${ }^{8-10}$.

\section{Entrevista estructurada de organización de personalidad (STIPO)}

La STIPO es una entrevista semiestructurada, que consta de 87 preguntas en 6 Dimensiones
(Identidad, Calidad de Relaciones Objetales, Defensas Primitivas, Afrontamiento/Rigidez, Agresión y Valores Morales) las que permiten evaluar la Organización de la Personalidad en base a conceptos psicoanalíticos ${ }^{7}$.

La STIPO evalúa la Identidad a través de la capacidad del individuo para investir el trabajo, estudios y/o actividades de tiempo libre y el grado de integración y estabilidad del sentido de sí mismo, incluyendo la estabilidad y valencia general de la autoestima. La Identidad es también apreciada a través de la evaluación del sentido que tiene el sujeto de otros, el grado de integración y estabilidad en la vivencia de los otros, junto con la capacidad de evaluar a los otros en profundidad y para percibir en forma precisa lo que los otros están sintiendo y pensando ${ }^{7}$.

\section{Metodología}

El diseño de investigación se basó en un enfoque cuantitativo, no experimental de tipo transversal. La muestra correspondió a 61 personas, dividida en 2 grupos muestrales de pacientes del Hospital Psiquiátrico del Salvador de Valparaíso, grupo A con diagnóstico de Trastornos de Personalidad según criterios del DSM-IV (30 personas) y grupo B sin Trastornos de Personalidad (31 personas). Fue una muestra intencionada, seleccionando a los participantes en base a criterios de inclusión y de exclusión predeterminados por los investigadores.

\section{Instrumento de medición}

Este estudio aplicó la STIPO en su Dimensión Identidad con sus 29 preguntas.

- Capacidad de Investir (preguntas 1 a 11).

- Sentido de Sí mismo-Coherencia y Continuidad (preguntas 12 a 20) y

- Sentido de los Otros (preguntas 21 a 29).

El sistema de puntuación va de una escala de 0 a 2, donde 0 refleja la ausencia de patología de acuerdo a lo que esté midiendo la escala; el puntaje 1 representa un estado intermedio de patología y 2 refleja una clara presencia de patología de acuerdo a la característica que está siendo evaluada ${ }^{7-10}$. 
La participación de los pacientes fue voluntaria y sin costo económico, con firma de consentimiento informado. La privacidad de los participantes ha sido respetada mediante la confidencialidad de los investigadores omitiéndose el nombre de los participantes y asignándose un número a cada uno de ellos.

\section{Resultados}

Una vez aplicados los criterios de inclusión y de exclusión, cada investigador seleccionó la mitad de la muestra correspondiente a pacientes con diagnóstico previo, realizado por psiquiatras en ficha clínica y fue derivado al otro investigador a fin de aplicar la STIPO sin dar ningún antecedente diagnóstico.

De esta forma se entrevistaron 61 personas, 30 personas con diagnóstico de Trastorno Personalidad (grupo A) según DSM-IV y 31 personas sin Trastorno de Personalidad según DSM-IV (grupo B) $)^{11}$ (Tabla 1).

\section{Resultados de la entrevista STIPO}

Se obtuvo un total de 31 pacientes con presencia de Difusión de Identidad y un total de 30 pacientes con Identidad Integrada.

El patrón de comportamiento de cada pregunta para el grupo A (Figura 1), presenta un claro pre- dominio en la presencia de patología (categoría 2) a partir de la pregunta 9, con porcentajes por ítems entre un $41 \%$ y un $87 \%$ de individuos con presencia de patología y que en promedio representan el $74 \%$, seguido de un estado intermedio (categoría 1) con un $22 \%$ y solo un $4 \%$ para la categoría ausencia de patología (categoría 0 ).

El grupo $\mathrm{B}$, mostró un claro predominio en la ausencia de patología (categoría 0) para casi todas las preguntas, con porcentajes por ítems que fluctúan entre un $45 \%$ y un $94 \%$ de individuos con ausencia de patología, seguido de un estado intermedio (categoría 1) con un $29 \%$ y solo un $5 \%$ para la categoría presencia de patología (categoría 2) (Figura 2).

\section{Nivel de concordancia entre vías diagnósticas}

Se obtuvo un alto grado de concordancia, siendo de un 100\% entre el diagnóstico de "Difusión de Identidad" de la prueba STIPO y el diagnóstico Trastorno de Personalidad según el DSM-IV y de un $97 \%$ entre el diagnóstico de "Identidad Integrada" de la prueba STIPO y el diagnóstico sin Trastorno de Personalidad en la DSM-IV.

\section{Comparación entre grupos para cada ítem}

Con la utilización de la prueba $U$ de MannWhitney para probar diferencias en el comporta-

Tabla l. Tamaños de muestra para grupo A y B, según edad, sexo y estado civil

\begin{tabular}{|c|c|c|c|c|c|c|c|c|c|c|c|c|c|c|}
\hline \multirow[t]{2}{*}{ Grupos } & \multirow[t]{2}{*}{ Edad } & \multicolumn{6}{|c|}{ Mujeres } & \multicolumn{6}{|c|}{ Hombres } & \multirow[t]{2}{*}{ Total } \\
\hline & & Cas. & Con. & Div. & Sep. & Solt. & Total & Cas.. & Con & Div. & Sep. & Solt. & Total & \\
\hline \multirow[t]{4}{*}{ A } & 18 a 30 & 2 & 1 & - & 1 & 7 & 11 & - & - & - & - & 1 & 1 & 12 \\
\hline & 31 a 40 & 1 & 1 & - & 3 & - & 5 & - & - & - & 1 & 1 & 2 & 7 \\
\hline & 41 a 57 & 5 & 1 & 1 & 2 & - & 9 & - & 1 & - & - & 1 & 2 & 11 \\
\hline & Total & 8 & 3 & 1 & 6 & 7 & 25 & - & 1 & - & 1 & 3 & 5 & 30 \\
\hline \multirow[t]{4}{*}{ B } & 18 a 30 & 1 & 1 & - & 1 & 7 & 10 & - & - & - & - & 2 & 2 & 12 \\
\hline & 31 a 40 & 4 & - & - & - & 3 & 7 & 2 & - & - & 2 & 1 & 5 & 12 \\
\hline & 41 a 57 & 2 & 1 & - & 2 & - & 5 & 1 & - & - & 1 & - & 2 & 7 \\
\hline & Total & 7 & 2 & - & 3 & 10 & 22 & 3 & - & - & 3 & 3 & 9 & 31 \\
\hline Total & & 15 & 5 & 1 & 9 & 17 & 47 & 3 & 1 & 0 & 4 & 6 & 14 & 61 \\
\hline
\end{tabular}

Cas: Casado/a. Con: Conviviente. Div: Divorciado/a. Sep: Separado/a. Solt: Soltero/a. 


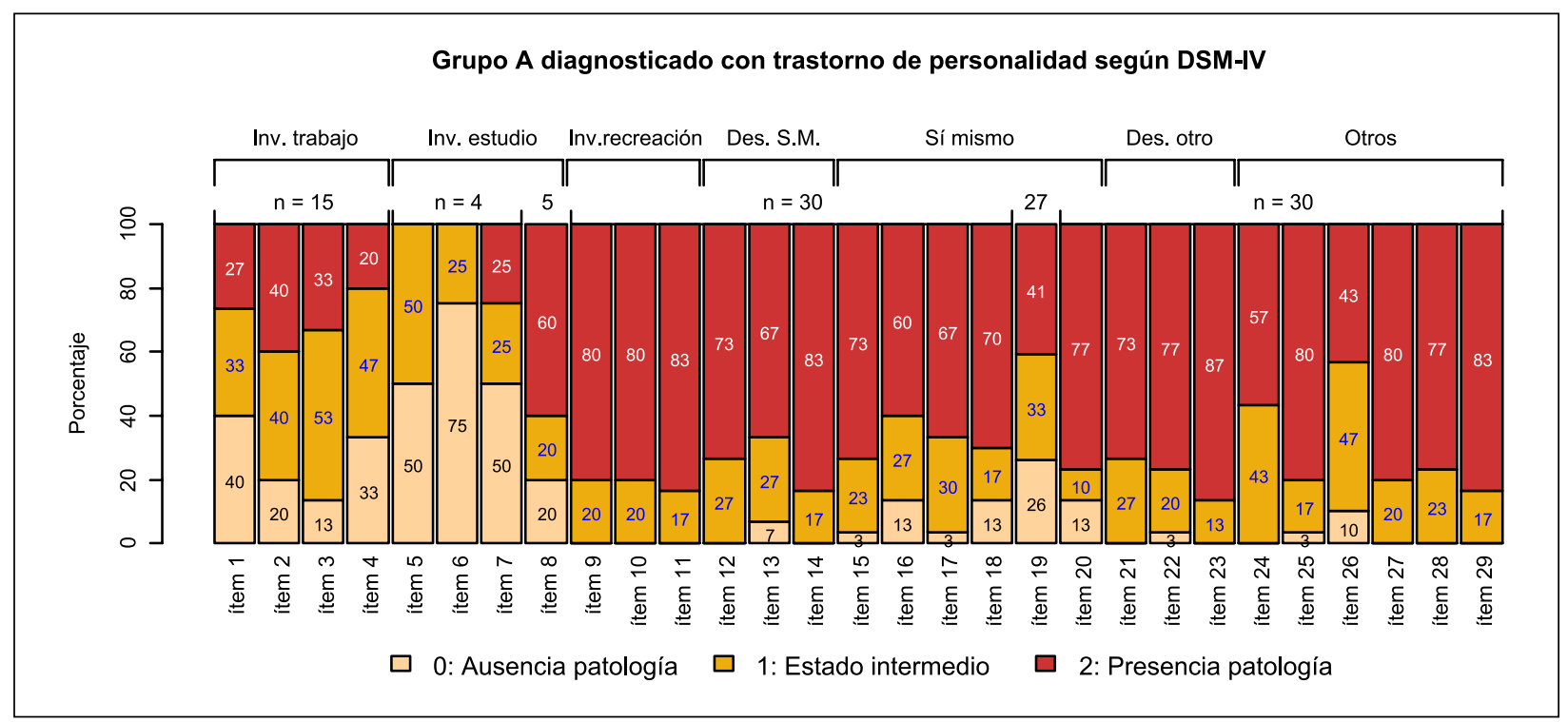

Figura 1. Gráfico de porcentajes apilados para la escala de 0 a 2 por cada ítem aplicado al grupo de individuos con diagnóstico de Trastorno de Personalidad según DSM-IV. Valores al interior de cada barra indican el porcentaje de representación en ese ítem de cada una de las subdimensiones.

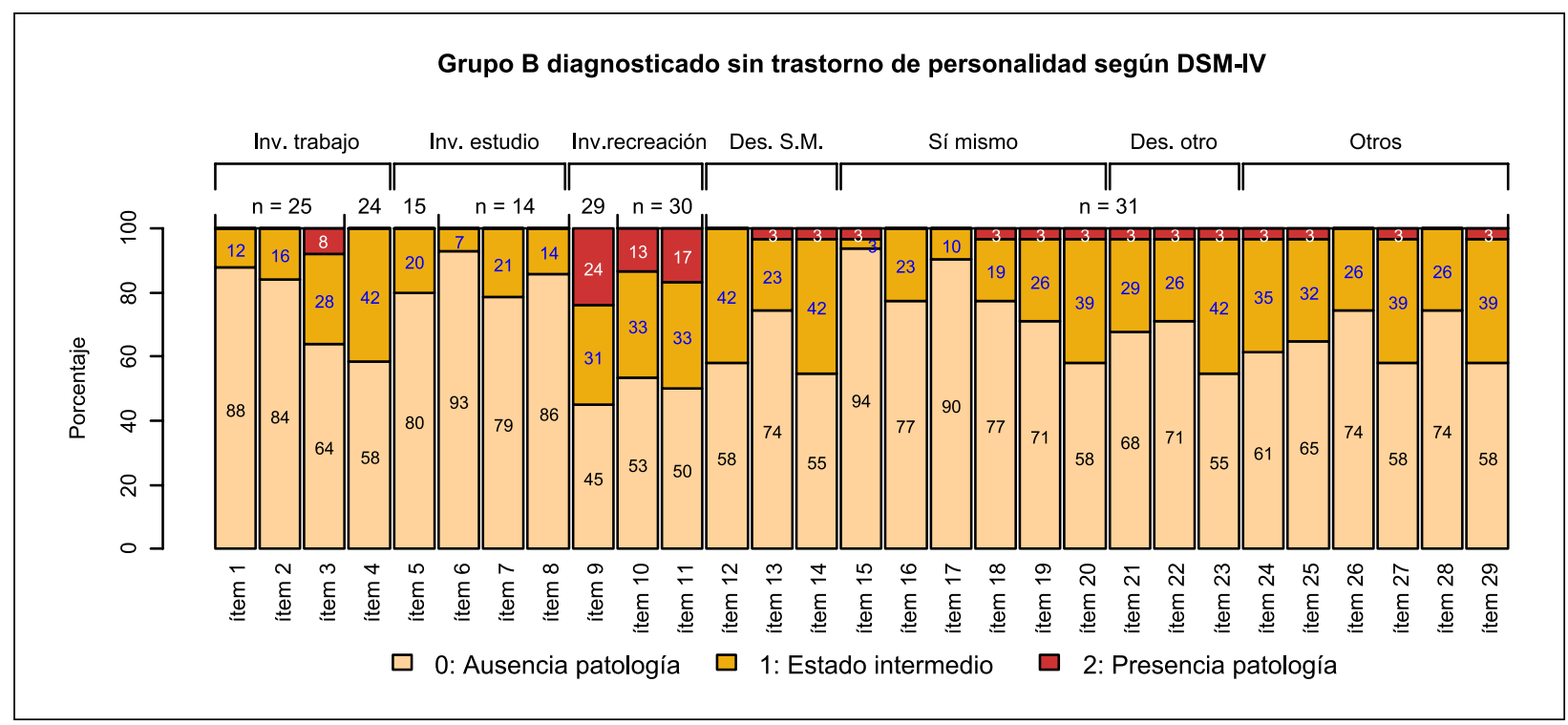

Figura 2. Gráfico de porcentajes apilados para la escala de 0 a 2 por cada ítem aplicado al grupo de individuos sin diagnóstico de trastorno de personalidad según DSM-IV. Valores al interior de cada barra indican el porcentaje de representación en ese ítem de cada una de las subdimensiones.

miento de las respuestas entre grupos, los resultados indicaron diferencias significativas entre los grupos por pregunta (valor $\mathrm{p}<0,05$ ) para la mayoría de los ítems, a excepción de las preguntas 4 a 7 influen- ciadas principalmente por los bajos tamaños de muestra presentes en el grupo A y que indican una nula o baja frecuencia de individuos con presencia de patología en esos ítems (valor $\mathrm{p}<0,05$, Tabla 2). 
Tabla 2. Prueba de normalidad por ítem, grupo y prueba de homogeneidad de varianzas y U de Mann-Whitney por ítem para los grupos de individuos clasificados con o sin trastorno de personalidad según DSM-IV

\begin{tabular}{|c|c|c|c|c|c|c|c|c|}
\hline \multirow[t]{2}{*}{ Ítems } & \multicolumn{4}{|c|}{$\begin{array}{l}\text { Prueba de normalidad de Shapiro Wilks } \\
\begin{array}{ll}\text { G. } A & \text { G. B }\end{array}\end{array}$} & \multicolumn{2}{|c|}{$\begin{array}{l}\text { Prueba Homegeneidad } \\
\text { de varianza de Bartlett }\end{array}$} & \multicolumn{2}{|c|}{$\begin{array}{c}\text { Prueba U de } \\
\text { Mann-Whitney }\end{array}$} \\
\hline & Estadístico & Valor $\mathbf{p}$ & Estadístico & Valor $\mathbf{p}$ & Estadístico & Valor p & Estadístico & Valor $\mathbf{p}$ \\
\hline Ítem 1 & 0,799 & $<0,05$ & 0,384 & $<0,05$ & 14,992 & $<0,05$ & 283,50 & $<0,05$ \\
\hline Ítem 2 & 0,806 & $<0,05$ & 0,445 & $<0,05$ & 9,494 & $<0,05$ & 319,50 & $<0,05$ \\
\hline Ítem 3 & 0,801 & $<0,05$ & 0,679 & $<0,05$ & 0,026 & 0,873 & 292,00 & $<0,05$ \\
\hline Ítem 4 & 0,817 & $<0,05$ & 0,629 & $<0,05$ & 2,663 & 0,103 & 240,00 & 0,055 \\
\hline Ítem 5 & 0,729 & $<0,05$ & 0,499 & $<0,05$ & 0,561 & 0,454 & 39,00 & 0,266 \\
\hline Ítem 7 & 0,630 & $<0,05$ & 0,297 & $<0,05$ & 2,144 & 0,143 & 33,00 & 0,381 \\
\hline Ítem 7 & 0,863 & 0,272 & 0,516 & $<0,05$ & 3,752 & 0,053 & 37,50 & 0,223 \\
\hline Ítem 8 & 0,771 & $<0,05$ & 0,428 & $<0,05$ & 5,629 & $<0,05$ & 61,00 & $<0,05$ \\
\hline Ítem 9 & 0,492 & $<0,05$ & 0,776 & $<0,05$ & 12,790 & $<0,05$ & 717,00 & $<0,05$ \\
\hline Ítem 10 & 0,492 & $<0,05$ & 0,745 & $<0,05$ & 8,988 & $<0,05$ & 798,00 & $<0,05$ \\
\hline Ítem 11 & 0,452 & $<0,05$ & 0,761 & $<0,05$ & 12,723 & $<0,05$ & 787,50 & $<0,05$ \\
\hline Ítem 12 & 0,554 & $<0,05$ & 0,629 & $<0,05$ & 0,344 & 0,558 & 878,00 & $<0,05$ \\
\hline Ítem 13 & 0,656 & $<0,05$ & 0,585 & $<0,05$ & 0,755 & 0,385 & 845,00 & $<0,05$ \\
\hline Ítem 14 & 0,452 & $<0,05$ & 0,704 & $<0,05$ & 4,672 & $<0,05$ & 880,00 & $<0,05$ \\
\hline Ítem 15 & 0,594 & $<0,05$ & 0,268 & $<0,05$ & 2,585 & 0,108 & 892,00 & $<0,05$ \\
\hline Ítem 16 & 0,705 & $<0,05$ & 0,519 & $<0,05$ & 8,159 & $<0,05$ & 826,00 & $<0,05$ \\
\hline Ítem 17 & 0,648 & $<0,05$ & 0,340 & $<0,05$ & 10,416 & $<0,05$ & 899,50 & $<0,05$ \\
\hline Ítem 18 & 0,622 & $<0,05$ & 0,550 & $<0,05$ & 3,444 & 0,063 & 823,50 & $<0,05$ \\
\hline Ítem 19 & 0,790 & $<0,05$ & 0,614 & $<0,05$ & 4,653 & $<0,05$ & 646,50 & $<0,05$ \\
\hline Ítem 20 & 0,548 & $<0,05$ & 0,693 & $<0,05$ & 1,591 & 0,207 & 809,50 & $<0,05$ \\
\hline Ítem 21 & 0,554 & $<0,05$ & 0,640 & $<0,05$ & 1,177 & 0,278 & 875,00 & $<0,05$ \\
\hline Ítem 22 & 0,559 & $<0,05$ & 0,614 & $<0,05$ & 0,041 & 0,839 & 868,50 & $<0,05$ \\
\hline Ítem 23 & 0,404 & $<0,05$ & 0,704 & $<0,05$ & 6,921 & $<0,05$ & 887,00 & $<0,05$ \\
\hline Ítem 24 & 0,632 & $<0,05$ & 0,679 & $<0,05$ & 0,367 & 0,544 & 837,00 & $<0,05$ \\
\hline Ítem 25 & 0,518 & $<0,05$ & 0,661 & $<0,05$ & 0,304 & 0,581 & 867,00 & $<0,05$ \\
\hline Ítem 26 & 0,771 & $<0,05$ & 0,547 & $<0,05$ & 4,452 & $<0,05$ & 815,50 & $<0,05$ \\
\hline Ítem 27 & 0,492 & $<0,05$ & 0,693 & $<0,05$ & 3,158 & 0,076 & 876,00 & $<0,05$ \\
\hline Ítem 28 & 0,526 & $<0,05$ & 0,547 & $<0,05$ & 0,032 & 0,857 & 902,00 & $<0,05$ \\
\hline Ítem 29 & 0,452 & $<0,05$ & 0,693 & $<0,05$ & 4,598 & $<0,05$ & 882,50 & $<0,05$ \\
\hline IG & 0,804 & $<0,05$ & 0,784 & $<0,05$ & 0,053 & 0,817 & 903,50 & $<0,05$ \\
\hline SSM & 0,793 & $<0,05$ & 0,661 & $<0,05$ & 2,021 & 0,155 & 927,50 & $<0,05$ \\
\hline SCO & 0,753 & $<0,05$ & 0,704 & $<0,05$ & 0,168 & 0,682 & 929,00 & $<0,05$ \\
\hline PGI & 0,597 & $<0,05$ & 0,688 & $<0,05$ & 3,099 & 0,078 & 925,00 & $<0,05$ \\
\hline
\end{tabular}

Valores p menores al 5\% indican rechazo de la hipótesis nula. G. A: grupo con trastorno de personalidad. G. B: grupo sin trastorno de personalidad. IG: investidura en general. SSM: sentido de sí mismo. SCO: sentido de los otros. PGI: puntuación general identidad. 


\section{Conclusiones}

En nuestra muestra las personas con Trastorno Severos de la Personalidad (grupo A) arrojaron los valores más altos en la entrevista STIPO en su Dimensión Identidad lo que determina una clara presencia de patología en la Identidad del Yo, vale decir, que presentaron Difusión de Identidad y, por otra parte, el grupo sin Trastorno de la Personalidad (grupo B) obtuvo los valores más bajos en la STIPO en su Dimensión Identidad lo que se traduce como Identidad Del Yo Integrada.

El comportamiento de las respuestas, para cada ítem por grupo, entregó evidencia a favor de la hipótesis de que es posible diagnosticar Difusión de Identidad o Identidad Integrada a través de la aplicación de la Dimensión Identidad de la Entrevista STIPO. No obstante, existe incertidumbre en algunos resultados particulares asociados a ciertas preguntas en la sub-Dimensión Capacidad de Investir dado el reducido tamaño de respuestas presente en los ítems 4 a 7.

Encontramos una gran concordancia entre el diagnóstico de Difusión de Identidad, obtenido a través de la STIPO (Dimisión Identidad), y el diagnóstico previo de Trastorno de Personalidad según el DSM-IV. Así mismo encontramos una gran concordancia entre el diagnóstico de Identidad Integrada obtenido a través de la STIPO (Dimensión Identidad) y la ausencia de Trastorno de Personalidad evidenciable según el DSM-IV. Por lo tanto, en esta investigación encontramos un alto grado de concordancia entre estas dos diferentes vías diagnósticas, que, sin embargo, guardan íntima relación clínica como ya se ha referido. En este trabajo la Evaluación de la Identidad resultó con un alto peso estadístico y clínico como elemento diagnóstico específico y común de pacientes que agrupamos bajo el título de “Trastornos Severos De La Personalidad" o pacientes con Organización De La Personalidad Limítrofe como los denomina Kernberg.

Respecto a las limitaciones del presente trabajo de investigación podemos señalar que el tamaño de la muestra fue reducido, de una población específi$\mathrm{ca}$, lo que no permite que se puedan generalizar ni a la población psiquiátrica ni a la población general nacional.

Además, señalamos que ambos investigadores participamos en la selección final de la muestra y la aplicación de la STIPO, lo cual podría incidir en un eventual sesgo.

En nuestra muestra estudiada no tuvimos dificultades a la hora de calificar presencia de Difusión de Identidad o Identidad Integrada lo cual probablemente se debió a que la muestra fue intencionada, de tal manera que no se obtuvieron puntajes intermedios (Puntaje 3) sino, más bien, extremos. Sería interesante evaluar muestras con resultados intermedios, esto tomando en cuenta que hasta el momento no existe un punto de corte de promedios definido por los autores de la STIPO que permita determinar la presencia o ausencia de Difusión de Identidad.

Tomando en cuenta los resultados obtenidos en esta investigación, podemos señalar que la entrevista STIPO, en su Dimensión Identidad, resultó altamente coincidente el tener Identidad Integrada con el no presentar Trastornos Severos de la Personalidad tipo DSM y, por otra parte, altamente coincidente el presentar Difusión de Identidad y tener alguno de los diagnósticos de Trastornos Severos de la Personalidad según el DSM.

Teniendo en mente el modelo de Kernberg y la inclusión de Evaluación de la Identidad en el DSM5 por ahora contenida en la sección III $^{12}$, así como la opinión de otros autores contemporáneos ${ }^{13-18}$, nuestro estudio pretendió enfatizar la importancia de incorporar la evaluación de la Identidad del Yo a la evaluación clínica tradicional, por cuanto es un elemento fundamental, prioritario y discriminatorio que permite determinar el tipo de Organización de Personalidad entre Neurótico y Limítrofe, que dada su relevancia debe ser incorporado en los procesos de diagnósticos tradicionales. 


\section{Resumen}

Estudio que aplicó la escala STIPO (Structured Interview of Personality Organization - Entrevista Estructurada de Organización de Personalidad) en su Dimensión Identidad a 61 pacientes cony sin diagnóstico previo de Trastorno de Personalidad según DSM-IV, para evaluar el estado de la Identidad del Yo. Esta investigación se basó en el Modelo de Otto Kernberg y colaboradores, quienes consideran que la Identidad del Yo es el criterio central a la hora de evaluar la Organización de la Personalidad. Este trabajo expondrá los principales fundamentos teóricos y clínicos en referencia a la Identidad del Yo de este autor. En esta muestra, encontramos un alto grado de concordancia entre el diagnóstico de Trastorno de Personalidad según DSM y la presencia de Difusión de Identidad a través de la STIPO Dimensión Identidad y, por otra parte, Identidad Integrada en pacientes que no presentaron Trastorno de Personalidad según DSM.

Palabras clave: Identidad, diagnóstico estructural, trastorno de la personalidad.

\section{Referencias bibliográficas}

1. American Psychiatric Association. Diagnostic and statistical manual of mental disorders. 4th ed. Washington (DC): American Psychiatric Association. 1994.

2. Identity: Recent Findings and Clinical. Kernberg, O. The Psychoanalytic Quarterly 2006; 75: 9691003.

3. Kernberg O. Object Relations Theory, and Clinical Psychoanalysis. New York: Jason Aronson. 1976.

4. Kernberg O. Trastornos Graves de la Personalidad: Estrategias Psicoterapéuticas. México D.F.: Editorial El Manual Moderno. 1984.

5. Caligor E, Kernberg O, Clarkin J. Handbook of dynamic psychotherapy for higher level personality pathology. 2007.

6. Yeomans F, Clarkin J, Kernberg O. Psicoterapia centrada en la transferencia, su aplicación al trastorno límite de la personalidad. Manual Clínico para profesionales. Editorial Desclee De Bruwer, S.A. 2016

7. Clarkin J, Caligor E, Stern B, Kernberg O. Structured interview for personality organization (STIPO). White Plains, NY: The Personality Disorders Institute. Weill College of Medicine of Cornell University. 2006.

8. Stern B, Caligor E, Clarkin J, Critchfield K, Horz $\mathrm{S}$, Maccornack V, et al. Structured Interview of
Personality-Organization (STIPO): Preliminary Psychometrics in a Clinical Sample. Journal of Personality Assessment 2010; 92 (1): 35-44.

9. Balbontin AM, Isaac A, Pollak H. Traducción y validación del Instrumento Structured interview of personality organization (STIPO): Dimensión Identidad. Tesis para optar al grado académico de magíster. Universidad del Desarrollo, Santiago, Chile. 2008.

10. Doering S, Burgmer M, Heuft G, Menke D, Bäumer B, Lübking $\mathrm{M}$, et al. Reliability and validity of the German version of the Structured Interview of Personality Organization (STIPO). BMC Psychiatry 2013; 13: 210. doi: 10.1186/1471-244X-13-210.

11. Kernberg O. Structural interviewing. Psychiatric Clinics of North America 1981; 4 (1): 169-95.

12. American Psychiatric Association. Diagnostic and statistical manual of mental disorders. 5 ed. Washington (DC): American Psychiatric Association. 2013.

13. Garnet RE, Levy KN, Mattanah JJF, Edell WS, Mcglashan TH. Borderline personality disorder in adolescence: ubiquitous or specific? Amer J Psychiatry 1994; 151: 1380-2.

14. Wilkinson-Ryan T, Westen D. Identity disturbance in borderline personality disorder: An empirical investigation. American Journal of Psychiatry 2000; 157: 528-41.

15. Levy K. The implications of attachment theory 
and research for understanding borderline personality disorder. Development and Psychopathology 2005; 7959-986.

16. Westen D, Betan E, Defife J. Identity disturbance in adolescence: Associations with borderline personality disorder. Dev Psychopathol 2011; 23: 305-13.

17. Jung E, Pick O, Schlüter-Müller S, Schmeck K, Goth K. Identity development in adolescents with mental problems. Child and Adolescent Psychiatry and Mental Health 2013; 7: 26.

18. Goth K, Foelsch P, Schlüter-Müller S, Birkhölzer M, Jung E, Pick O, et al. Assessment of identity development and identity diffusion in adolescence-Theoretical basis and psychometric properties of the self-report Questionnaire AIDA. Child and Adolescent Psychiatry and Mental Health 2012; 6: 27 doi:10.1186/1753-2000-6.

Correspondencia:

Dr. Roberto Castillo Tamayo

Edificio Blanca Estela 60 oficina 51

Bosques de Montemar-Concón

rcastillot5@gmail.com 